\title{
Journal of Bone and Mineral Metabolism Best Paper Award 2021
}

(c) The Japanese Society Bone and Mineral Research 2021

The Journal of Bone and Mineral Metabolism Best Paper Award was established in 2008. Candidates for the award must be members of the Japanese Society of Bone and Mineral Research, and the winner is honored at the Society's Annual Meeting.

We are pleased to announce that the following article has received JBMM Best Paper Award.

"Decreased sensory nerve excitation and bone pain associated with mouse Lewis lung cancer in TRPV1-deficient mice"

by

Hiroki Wakabayashi, Satoshi Wakisaka, Toru Hiraga, Kenji Hata, Riko Nishimura, Makoto Tominaga, Toshiyuki Yoneda

J Bone Miner Metab (2018) 36:274-285

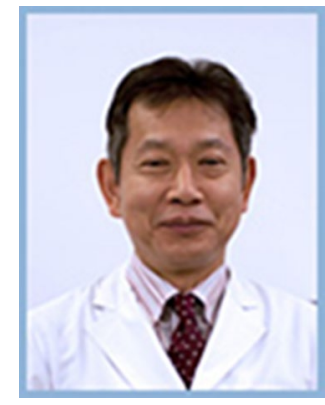

Dr. Hiroki Wakabayashi

\begin{abstract}
Bone pain is one of the most common and life-limiting complications of cancer metastasis to bone. Although the mechanism of bone pain still remains poorly understood, bone pain is evoked as a consequence of sensitization and excitation of sensory nerves (SNs) innervating bone by noxious stimuli produced in the microenvironment of bone metastases. We showed that bone is innervated by calcitonin gene-related protein (CGRP) ${ }^{+} \mathrm{SNs}$ extending from dorsal root ganglia (DRG), the cell body of SNs, in mice. Mice intratibially injected with Lewis lung cancer (LLC) cells showed progressive bone pain evaluated by mechanical allodynia and flinching with increased CGRP ${ }^{+} \mathrm{SNs}$ in bone and augmented SN excitation in DRG as indicated by elevated numbers of pERK- and pCREB-immunoreactive neurons. Immunohistochemical examination of LLC-injected bone revealed that the tumor microenvironment is acidic. Bafilomycin A1, a selective inhibitor of $\mathrm{H}^{+}$secretion from vacuolar proton pump, significantly alleviated bone pain, indicating that the acidic microenvironment contributes to bone pain. We then determined whether the transient receptor potential vanilloid 1 (TRPV1), a major acid-sensing nociceptor predominantly expressed on SNs, plays a role in bone pain by intratibially injecting LLC cells in TRPV1-deficient mice. Bone pain and SN excitation in the DRG and spinal dorsal horn were significantly decreased in TRPV $1^{-1-}$ mice compared with wild-type mice. Our results suggest that TRPV1 activation on SNs innervating bone by the acidic cancer microenvironment in bone contributes to SN activation and bone pain. Targeting acid-activated TRPV1 is a potential therapeutic approach to cancer-induced bone pain.
\end{abstract}

We offer our sincere congratulations on behalf of the Journal of Bone and Mineral Metabolism, with best wishes for further development of the author's research.
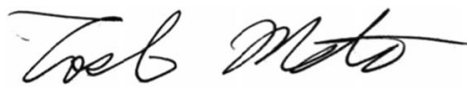

Toshio Matsumoto

Editor-in-Chief

Journal of Bone and Mineral Metabolism

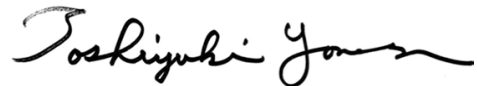

Toshiyuki Yoneda

Editor-in-Chief

Journal of Bone and Mineral Metabolism 